\title{
Black-box inhomogeneous preconditioning for self-consistent field iterations in density functional theory
}

\author{
Michael F. Herbst因 and Antoine Levit地 \\ Inria Paris and CERMICS, Ecole des Ponts, $6 \& 8$ avenue Blaise Pascal, 74455 Marne-la-Vallee, France.
}

\begin{abstract}
We propose a new preconditioner based on the local density of states for computing the selfconsistent problem in Kohn-Sham density functional theory. This preconditioner is inexpensive and able to cure the long-range charge sloshing known to hamper convergence in large, inhomogeneous systems such as clusters and surfaces. It is based on a parameter-free and physically motivated approximation to the independent-particle susceptibility operator, appropriate for both metals and insulators. It can be extended to semiconductors by using the macroscopic electronic dielectric constant as a parameter in the model. We test our preconditioner successfully on inhomogeneous systems containing metals, insulators, semiconductors and vacuum.
\end{abstract}

\section{INTRODUCTION}

Since its original formulation in the $1960 s^{112}$ KohnSham density-functional theory (DFT) has become one of the most widespread methods for simulating properties in solid-state physics and chemistry. Its advantageous balance balance between accuracy and computational cost as well as the continuous development of more efficient methods ${ }^{3}$ has continuously increased its range of applicability. Nowadays DFT-based $a b$ initio treatments are standard in many fields 45 . Driven by the availability of ever-growing amounts of computational power, several groups have developed high-throughput DFT frameworks where the properties of a large numbers of sys-

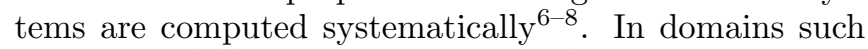
as catalysis ${ }^{9} \sqrt{12}$ or battery research $\frac{13}{}$, where systematic experiments are expensive or time-consuming, large-scale screening studies represent an interesting alternative to computationally discover novel compounds or boil down a large candidate list to a tractable set for more detailed follow-up investigation. Compared to the early years where the focus was on performing a small number of computations on hand-picked systems, high-throughput studies have much stronger requirements. In particular any form of manual parameter selection is not practical. The typical approach to tackle this problem is to provide a set of heuristics, which automatically select the computational parameters based on prior experience $e^{14}$. This empirical process is, however, problematic, because resulting methods can still fall short or break down in cases with unexpected behavior, amongst which one often finds exactly the interesting cases such studies were hoped to excavate in the first place. A clear objective for improving DFT algorithms is therefore to employ as small a number of parameters as possible, ideally leading to a simulation workflow being composed only of blackbox building blocks that make use of the appropriate physics to adapt to the system at hand.

With this idea in mind, this work will consider the self-consistent field (SCF) problem as a fundamental step in all Kohn-Sham DFT simulations. As it is wellknown, simple SCF schemes suffer from a number of instabilities, which in the linear regime can be seen to arise from the properties of the dielectric operator $\varepsilon=1-\left(v_{c}+K_{\mathrm{XC}}\right) \chi_{0}$. This operator is built up from three terms: the independent-particle susceptibility $\chi_{0}$, the Coulomb kernel $v_{c}$ and the exchange-correlation kernel $K_{\mathrm{XC}}$. Based on an analysis of these three components, we classify the possible sources of slow convergence in three types: (a) systems close to an electronic phase transition, for instance ferromagnetic, due to the negativity of $K_{\mathrm{XC}}$; (b) localized $d$ or $f$ orbitals, giving large contributions to $\chi_{0}$; or (c) the long-range "chargesloshing" due to the divergence of the Coulomb operator $v_{c}$ at large wavelengths, especially prominent as larger systems are considered. The latter forms the main focus of this work. For an early discussion of these aspects, we refer to the work of Dederichs and Zeller 15 . In applications such as the ones mentioned above, all these sources of instabilities may occur and need to be accounted for by a preconditioner to yield fast and reliably converging SCF methods for high-throughput calculations. Moreover, in fields such as catalysis one is typically concerned with interfaces, surface effects or surface defects, i.e. situations in which simulations on large and/or inhomogeneous systems are to be performed.

Based on the successful application of the preconditioner suggested by Kerker ${ }^{16}$ for bulk metallic systems and (with minor modifications) for bulk semiconductors ${ }^{3}$, several attempts have been made to generalize Kerker's approach to inhomogeneous systems. For example, the work of Raczkowski et al. 17 discusses an approach by solving a Thomas-Fermi-von Weizsäcker problem at every SCF step. More recently, Zhou et al. ${ }^{18}$ suggested a more generalized parametrization of the Kerker model for mixed systems along with various strategies to adjust the respective parameters. A more general approach, solving a partial differential equation with variable coefficients, is the "elliptic preconditioner" by Lin and Yang $\frac{19}{}$. Related is the work by Hasnip and Probert 20 where SCF convergence is improved by mapping at each SCF step the Kohn-Sham problem to a simpler "auxiliary system" and solving that. Yet another alternative approach is to directly compute an approximate dielectric matrix based on the Kohn-Sham orbitals ${ }^{2122}$ and use this operator for preconditioning. We refer to Woods et al. ${ }^{[23}$ for a recent 
and thorough review of these approaches.

While these methods have been successfully applied to different classes of inhomogeneous systems like metallic slabs or clusters, none of them has so far proven to be both robust and general enough to prevent chargesloshing in all kinds of inhomogeneous systems. Additionally some of these methods can be either difficult to implement, too expensive to employ in big systems or require the selection of many or complicated system-specific parameters, making automated computations non-trivial. In this work, we present an inexpensive and easy to implement preconditioning strategy based on the local density of states. For systems involving only metals, insulators and vacuum, this preconditioner is parameter-free and thus completely black-box. Its range of applicability can be extended to systems involving semiconductors by introducing an additional parameter, the dielectric constant — which for most materials is a priori known. For all tested systems, we find that our preconditioner is able to cure long-range charge sloshing (cause (c) in the above classification), leaving only localized states and electronic phase transitions as sources of slow convergence to be investigated in future work.

The structure of this manuscript is as follows. Section II reviews the mathematical structure of the SCF problem in Kohn-Sham DFT and provides key results about rates of convergence, which are illustrated by example calculations in Section III. Section IV reviews common strategies for SCF preconditioning in bulk systems for which Section V provides computational results. Finally Sections VI and VII introduce the LDOS preconditioner in contrast to other approaches for preconditioning heterogeneous systems and presents an extensive study illustrating its performance in large mixed systems.

\section{SELF-CONSISTENT FIELD ITERATIONS}

Assume an isolated system of $2 N$ electrons with periodic boundary conditions in a (possibly nonlocal) pseudopotential $V_{\text {ext }}$ created by nuclei and core electrons. The Kohn-Sham equations for $N$ electron pairs at finite temperature $T$ are (in atomic units)

$$
\begin{aligned}
& \left(-\frac{1}{2} \nabla^{2}+V_{\mathrm{ext}}+V_{\mathrm{HXC}}(\rho)\right) \psi_{i}=\varepsilon_{i} \psi_{i} \\
& \rho=\sum_{i=1}^{\infty} f\left(\frac{\varepsilon_{i}-\varepsilon_{F}}{T}\right)\left|\psi_{i}\right|^{2} \\
& V_{\mathrm{HXC}}(\rho)=v_{c} \rho+V_{\mathrm{XC}}(\rho) \\
& \int \rho(\mathbf{r}) \mathrm{d} \mathbf{r}=2 N
\end{aligned}
$$

where $f(x)=2 /\left(1+e^{x}\right)$ is twice the Fermi-Dirac function, $V_{\mathrm{XC}}(\rho)$ is the exchange-correlation potential, and $v_{c} \rho$ is the Coulomb potential associated to the charge density $\rho$ in a uniform neutralizing background:

$$
-\nabla^{2}\left(v_{c} \rho\right)=4 \pi \rho
$$

We define the potential-to-density mapping $F$ by

$$
F(V)=\sum_{i=1}^{\infty} f\left(\frac{\varepsilon_{i}-\varepsilon_{F}}{T}\right)\left|\psi_{i}\right|^{2}
$$

where $\left(\varepsilon_{i}, \psi_{i}\right)$ are the eigenpairs of $-\frac{1}{2} \nabla^{2}+V_{\text {ext }}+V$ and the Fermi level $\varepsilon_{F}$ is uniquely defined by the condition

$$
\int(F(V))(\mathbf{r}) \mathrm{d} \mathbf{r}=2 N
$$

The Kohn-Sham equations can then be written as the fixed-point equation

$$
F\left(V_{\mathrm{HXC}}(\rho)\right)=\rho .
$$

The simplest self-consistent iteration is

$$
\rho_{n+1}=F\left(V_{\mathrm{HXC}}\left(\rho_{n}\right)\right) .
$$

Except on very simple systems, this iteration usually does not converge. A more useful scheme is the damped iteration

$$
\rho_{n+1}=\rho_{n}+\alpha\left[F\left(V_{\mathrm{HXC}}\left(\rho_{n}\right)\right)-\rho_{n}\right] \text {, }
$$

where $\alpha>0$ is a damping parameter. Note that we consider in this paper density mixing algorithms, but all our results are easily transposable to potential mixing.

It is very instructive to consider the convergence behavior of this iteration near a fixed-point $\rho_{*}$. As is wellknown, the iteration converges locally if and only if all the eigenvalues of the Jacobian matrix

$$
J_{\alpha}=1-\alpha\left(1-\chi_{0} K\right)
$$

have magnitude less than one. In this case, the convergence rate is equal to the eigenvalue of largest magnitude of $J_{\alpha}$. In this equation,

$$
\chi_{0}=F^{\prime}\left(V_{\mathrm{HXC}}\left(\rho_{*}\right)\right)
$$

is the independent-particle susceptibility, and

$$
K=V_{\mathrm{HXC}}^{\prime}\left(\rho_{*}\right)=v_{c}+K_{\mathrm{XC}}\left(\rho_{*}\right)
$$

is the derivative of the Hartree-exchange-correlation potential (the Hartree-exchange-correlation kernel).

Note that $J_{\alpha}=1-\alpha \varepsilon^{\dagger}$, where

$$
\varepsilon^{\dagger}=1-\chi_{0} K
$$

is the adjoint of the dielectric matrix $\varepsilon=1-K \chi_{0}$. As will be shown in Section IV, the susceptibility $\chi_{0}$ is a non-positive self-adjoint operator, and $K$ is self-adjoint. It follows that

$$
\chi_{0} K=-\left(-\chi_{0}\right)^{1 / 2}\left(-\chi_{0}\right)^{1 / 2} K
$$


has the same spectrum (except possibly for 0 ) than the symmetrized self-adjoint operator $\left(-\chi_{0}\right)^{1 / 2} K\left(-\chi_{0}\right)^{1 / 2}$, and therefore that $\varepsilon^{\dagger}$ has real spectrum.

It is often a good approximation to neglect the term $K_{\mathrm{XC}}=V_{\mathrm{XC}}^{\prime}\left(\rho_{*}\right)$ in $K$, yielding the random phase approximation (RPA)

$$
K_{\mathrm{RPA}}=v_{c}
$$

Since $v_{c}$ has non-negative spectrum, $\varepsilon^{\dagger}=1-\chi_{0} v_{c}$ has positive spectrum. If we do not assume the RPA approximation, $K$ does not have a guaranteed sign due to the non-convexity introduced in the model by the exchange-correlation term. The eigenvalues of $\chi_{0} K$ can thus be in principle arbitrary. It can, however, be shown that, at an energy local minimizer, $\varepsilon^{\dagger}$ has non-negative spectrum $15|24| 25$.

It follows that, near a non-degenerate energy minimizer, by selecting $\alpha$ small enough the spectrum of $J_{\alpha}=1-\alpha \varepsilon^{\dagger}$ will be in $(-1,1)$, and therefore the damping iteration (7) is locally convergent (see Cancès et al. ${ }^{25}$, Theorem 3.6). However, this convergence may be unacceptably slow in practice. Letting $\lambda_{\min }>0$ and $\lambda_{\max }$ be the lowest and largest eigenvalues of $\varepsilon^{\dagger}$, the convergence rate is $R=\max \left(\left|1-\alpha \lambda_{\min }\right|,\left|1-\alpha \lambda_{\max }\right|\right)$. Assuming that $\alpha$ is selected optimally to minimize this convergence rate, we obtain

$$
\alpha=\frac{2}{\lambda_{\min }+\lambda_{\max }}
$$

and

$$
R=\frac{\kappa-1}{\kappa+1}
$$

where

$$
\kappa=\frac{\lambda_{\max }}{\lambda_{\min }}
$$

is the (metric-independent) "spectral condition number". Note that this number is distinct from the usual (metricdependent) condition number of $\varepsilon^{\dagger}$, equal to the ratio of large to lowest singular values, because this operator is not self-adjoint. The spectral condition number $\kappa$ can however be identified with the condition number of $\varepsilon^{\dagger}$ under the metric induced by $-\chi_{0}$, for which $\varepsilon^{\dagger}$ is selfadjoint. With an abuse of language, we will use the term "condition number" to refer to the "spectral condition number" (16) throughout this work.

For $\kappa$ large enough, $R \approx 1-2 / \kappa$ and therefore the number of iterations $\frac{\text { tol }}{\log R}$ needed to achieve a fixed tolerance tol grows proportionally to $\kappa$. Slowly converging SCF iterations are therefore linked to large values of $\kappa$, originating from small and large eigenvalues of $\varepsilon^{\dagger}=1-\chi_{0}\left(v_{c}+K_{\mathrm{XC}}\right)$. In practice, this can be attributed to three different classes of instabilities, linked to large modes of $K_{\mathrm{XC}}, \chi_{0}$ and $v_{c}$ respectively: (a) The first case is when $\varepsilon^{\dagger}$ has small eigenvalues. This can only happen in systems with strong exchangecorrelation effects, because under the RPA all eigenvalues of $\varepsilon^{\dagger}$ are greater than 1 . This phenomenon is usually (but not necessarily) associated with symmetry breaking. To see this, consider ferromagnetic systems, where $\varepsilon^{\dagger}$ has negative eigenvalues at the spin-unpolarized state (a solution of the Kohn-Sham equations which is not an energy minimum). Paramagnetic systems close to a ferromagnetic transition will display spin-unpolarized energy minima with small but positive eigenvalues for $\varepsilon^{\dagger}$. The modes associated with the slow convergence are spin-polarization modes.

(b) A second source of instabilities are large eigenvalues of $\varepsilon^{\dagger}$ brought about by large modes of $\chi_{0}$. In solids, these modes can for instance be due to localized states, such as $d$ or $f$ electrons, close to the Fermi level. In this case the associated modes are localized around atoms.

(c) A final source are large eigenvalues of $\varepsilon^{\dagger}$ originating from the long-range divergence of the Coulomb interaction (large modes of $v_{c}$ ). Indeed, if $\widehat{\rho}(\mathbf{q})$ are the Fourier coefficients of $\rho$, then

$$
\widehat{\left(v_{c} \rho\right)}(\mathbf{q})=\frac{4 \pi \widehat{\rho}(\mathbf{q})}{|\mathbf{q}|^{2}}
$$

which diverges for small q (long wavelengths). Depending on whether this effect is compensated or not by $\chi_{0}$, this can manifest as large eigenvalues of $\varepsilon^{\dagger}$. As we will see, no compensation takes place for metals in large supercells. In this case, the condition number increases with the square of the system size length. Associated modes are long-wavelength transfer of charge from one end of the system to another ("charge sloshing").

Among these three sources of instabilities, the third one is possibly the most serious, because there the convergence degrades with system size.

In this paper, we focus on curing this source of slow convergence using preconditioning. Preconditioning consists in replacing (7) by

$$
\rho_{n+1}=\rho_{n}+\alpha P^{-1}\left(F\left(V_{\mathrm{HXC}}\left(\rho_{n}\right)\right)-\rho_{n}\right)
$$

where $P$ is a preconditioner. The Jacobian of this method at convergence $\left(\rho=\rho_{*}\right)$ is

$$
J_{\alpha P}=1-\alpha P^{-1} \varepsilon^{\dagger} .
$$

It follows that the optimal preconditioner $P$ is simply the adjoint dielectric operator $\varepsilon^{\dagger}$. Indeed, in this case, when $\alpha=1$, the iteration (18) turns into the standard Newton method: $J_{P}=0$, and the method converges in one step in the linear approximation. In practice however, inverting this explicitly (or computing its action on a vector) 
is too costly. Therefore it is desirable to find a preconditioner $P$ that can be inverted efficiently and such that $P^{-1}\left(1-K \chi_{0}\right)$ has a condition number close to 1 .

\section{ANDERSON ACCELERATION}

Before turning our attention to the problem of finding good preconditioners, we address the convergence behavior of acceleration techniques, which are commonly used to speed up SCF convergence, and which we will use in our tests.

Acceleration techniques treat the fixed-point equation (7) as a black box iteration, and use the history $\rho_{1}, \ldots, \rho_{n}$ in order to extrapolate a better $\rho_{n+1}$ than the one obtained by the simple (preconditioned) damping method (18). There are many variants of these, the most used being the method variously known as $\mathrm{Pu}$ lay/DIIS/Anderson mixing/acceleration, which we will refer to as Anderson acceleration. We refer to Woods et al. ${ }^{23}$ for an extensive review in the context of KohnSham DFT, and to Chupin et al. 26 and references within for a mathematical analysis of these acceleration techniques. In particular, in the linear regime and with infinite history, Anderson acceleration is known to be equivalent to the well-known GMRES method to solve linear equations. Assuming non-linear effects to be negligible, we can expect that Anderson acceleration inherits the improved convergence properties of Krylov methods $\$$. In particular, the convergence rate of the GMRES method is

$$
R=\frac{\sqrt{\kappa}-1}{\sqrt{\kappa}+1}
$$

and therefore the number of iterations grows only as the square root of $\kappa$. Furthermore, an important property of Krylov methods is that they converge to the exact solution in $N$ steps if the matrix has $N$ distinct eigenvalues ${ }^{27}$, and therefore we can expect Anderson acceleration to be very efficient in the case where eigenvalues are clustered.

In practice, Anderson methods are truncated to a finite history size, and the impact of the nonlinearity on the efficiency of the extrapolation is complex. Unlike with the simple damping method, the convergence behavior of Anderson acceleration can not be understood solely from the condition number $\kappa$, and the number of iterations does not always follow the law predicted by the rate 20 .

In Figure 1 we exhibit the error in energy for three representative cases, selected to illustrate the possible behaviors. We compute the extreme eigenvalues of $P^{-1} \varepsilon^{\dagger}$ at convergence, and use them to determine the optimal step using (14). For comparison, the plot also shows the convergence estimated from the rates 15 for damped iterations and 20) for Anderson acceleration. For details on the computational procedures, we refer to Sections $\mathrm{V}$ and VII.

In cases (a) and (c) the simple mixing method $(m=$ 0 ) converges with a rate consistent with the theoretical
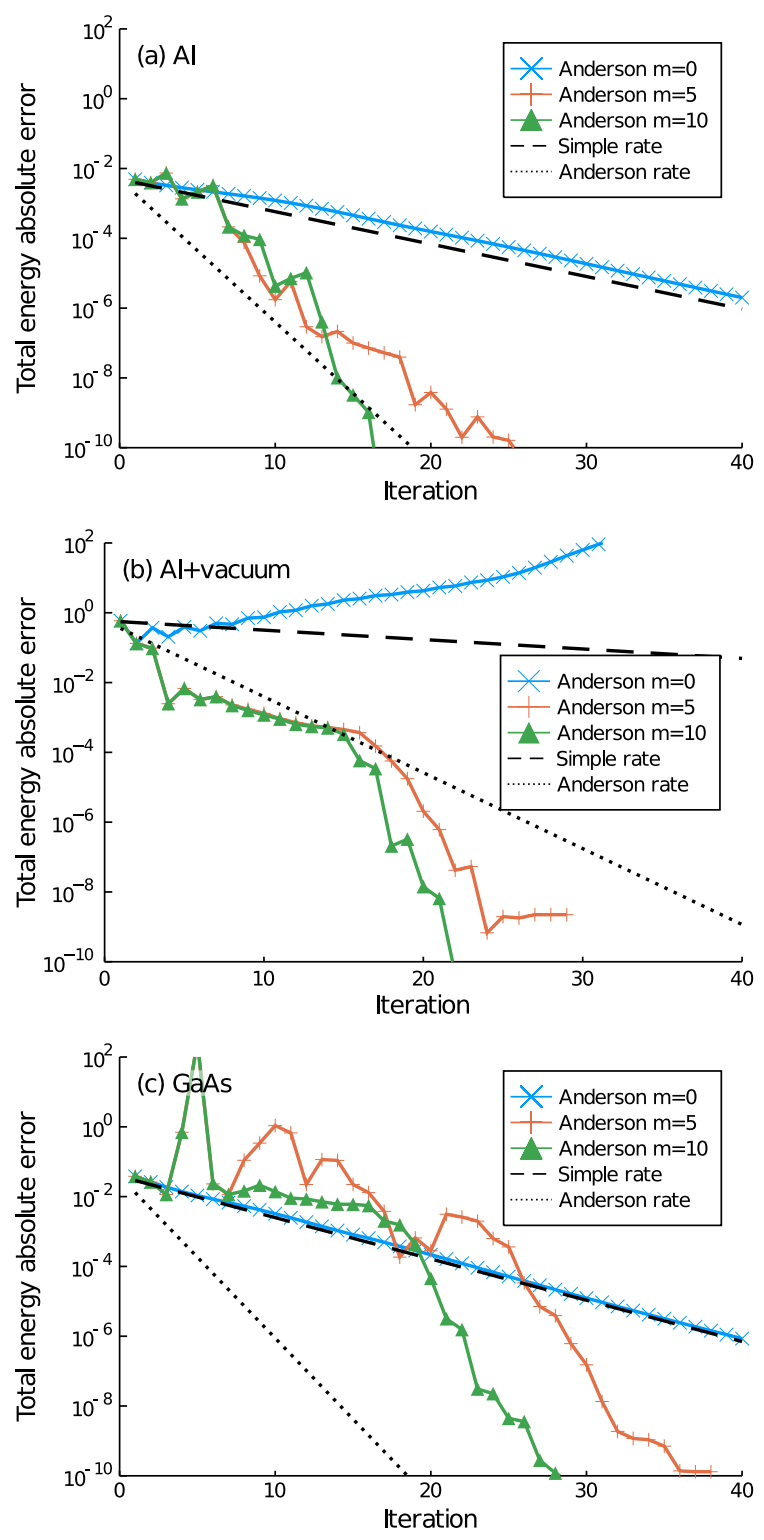

FIG. 1. Convergence of self-consistent field iterations on three selected systems using different history lengths $m$ for Anderson acceleration ( $m=0$ corresponds to simple mixing). From top to bottom the considered systems are (a) 12 layers of aluminium using a Kerker preconditioner; (b) 10 layers of aluminium with an equally wide portion of vacuum; and (c) 20 layers of gallium arsenide, in both latter cases using no preconditioner.

prediction. In case (b), using the optimal step computed at the solution proves too optimistic, and the iteration is divergent.

The accelerated methods track approximately the convergence rate (20) in case (a). Case (b) displays a characteristic "plateau" behavior: after an initial stage of rapid convergence, the error stagnates, then very rapid convergence is obtained once the accelerated scheme has "learned" the dominant clusters in the eigenvalues of 
$P^{-1} \varepsilon^{\dagger}$. The plateau is particularly pronounced in this case because $P^{-1} \varepsilon^{\dagger}$ has a very clustered spectrum: the largest eigenvalue is about 50 , but all eigenvalues except five are between 0.9 and 2.0. In case (c) the error shoots up abruptly around iterations 4 and 5 . This is because the linear model assumed by Anderson acceleration does not hold in the initial steps. In more complex cases, this can lead to complete divergence, but here the iteration recovers and then displays the plateau behavior seen in case (b). Notice that for $m=5$, where the history size is too small to capture all dominant clusters, the plateau has more the shape of a chain of half-arcs.

Overall one can conclude that while for non-accelerated fixed-point iterations the condition number and the resulting theoretical rates are very closely related to the observed number of iterations, the situation is less clear for the Anderson-accelerated procedure. In the following we will still employ Anderson acceleration for all our calculations for two reasons. Firstly because this represents the typical situation encountered in practice and secondly because otherwise, especially for cases with bad preconditioning, convergence can become extremely slow. To simplify our analysis we will also largely assume that the condition number of the preconditioned adjoint of the dielectric matrix $P^{-1} \varepsilon^{\dagger}$ is a good indicator for the expected number of iterations in the Anderson-accelerated $\mathrm{SCF}$, even though this discussion shows that the correlation is not as clear as one would expect from the simple estimate 20.

Note that, although the Anderson method is sometimes divergent, convergence can always be achieved (albeit very slowly) with the simple damping method by selecting a sufficiently small damping parameter. This means that it should be possible to stabilize the Anderson method by selecting stepsizes and extrapolation parameters more conservatively. This is a worthwhile research direction, but falls outside the scope of this paper.

\section{RESPONSE OPERATORS AND PRECONDITIONING}

\section{A. The independent-particle susceptibility $\chi_{0}$}

To examine the impact of the Coulomb divergence on the eigenvalues of the dielectric operator, we study the properties of the independent-particle susceptibility operator $\chi_{0}$ in periodic systems. Consider a perfect crystal with lattice $\mathcal{R}$, reciprocal lattice $\mathcal{R}^{*}$, Brillouin zone $\mathcal{B}$, unit cell $\Omega$ and $N_{\text {el }}$ electron pairs per unit cell. We choose a domain $\Omega_{\mathcal{N}}$, with periodic boundary conditions, composed of $\mathcal{N}=L_{1} \times L_{2} \times L_{3}$ copies of the unit cell of the crystal, and containing $\mathcal{N} N_{\text {el }}$ electron pairs. Then, as is standard, the eigenvectors of the periodic Hamiltonian $-\frac{1}{2} \nabla^{2}+V_{\text {ext }}+V_{\mathrm{HXC}}\left(\rho_{*}\right)$ can be chosen as the Bloch waves

$$
\psi_{n \mathbf{k}}(\mathbf{r})=\frac{1}{\sqrt{\mathcal{N}}} e^{i \mathbf{k} \cdot \mathbf{r}} u_{n \mathbf{k}}(\mathbf{r})
$$

where $u_{n \mathbf{k}}$ is a $\mathcal{R}$-periodic function normalized as

$$
\begin{aligned}
\left\langle u_{n \mathbf{k}} \mid u_{m \mathbf{k}}\right\rangle_{\Omega_{\mathcal{N}}} & =\mathcal{N}\left\langle u_{n \mathbf{k}} \mid u_{m \mathbf{k}}\right\rangle_{\Omega} \\
& =\mathcal{N} \int_{\Omega} u_{n \mathbf{k}}^{*}(\mathbf{r}) u_{m \mathbf{k}}(\mathbf{r}) \mathrm{d} \mathbf{r} \\
& =\mathcal{N} \delta_{n m} .
\end{aligned}
$$

The index $\mathbf{k}$ runs over the discrete set $\mathcal{B}_{\mathcal{N}} \subset \mathcal{B}$, which contains the $\mathcal{N}$ wavevectors in the Brillouin zone that are compatible with the periodic boundary conditions on $\Omega_{\mathcal{N}}$.

We now derive an explicit sum-over-states expression for $\chi_{0}=F^{\prime}\left(V_{\mathrm{HXC}}\left(\rho_{*}\right)\right)$. We consider an infinitesimal perturbation $\delta V$, and compute the response $\delta \rho=\chi_{0} \delta V$. We get

$$
\delta \rho=\sum_{\mathbf{k} \in \mathcal{B}_{\mathcal{N}}} \sum_{n=1}^{\infty}\left(\delta f_{n \mathbf{k}}\left|\psi_{n \mathbf{k}}\right|^{2}+f_{n \mathbf{k}}\left(\delta \psi_{n \mathbf{k}}^{*} \psi_{n \mathbf{k}}+\psi_{n \mathbf{k}}^{*} \delta \psi_{n \mathbf{k}}\right)\right)
$$

with $f_{n \mathbf{k}}=f\left(\frac{\varepsilon_{n \mathbf{k}}-\varepsilon_{F}}{T}\right)$. From first-order perturbation theory,

$$
\begin{aligned}
\delta f_{n \mathbf{k}} & =\left(\left\langle\psi_{n \mathbf{k}} \mid \delta V \psi_{n \mathbf{k}}\right\rangle_{\Omega_{\mathcal{N}}}-\delta \varepsilon_{F}\right) f_{n \mathbf{k}}^{\prime} \\
\delta \psi_{n \mathbf{k}} & =\sum_{\mathbf{k}^{\prime} \in \mathcal{B}_{\mathcal{N}}} \sum_{m=1}^{\infty} \frac{\left\langle\psi_{m \mathbf{k}^{\prime}} \mid \delta V \psi_{n \mathbf{k}}\right\rangle_{\Omega_{\mathcal{N}}}}{\varepsilon_{n \mathbf{k}}-\varepsilon_{m \mathbf{k}^{\prime}}} \psi_{m \mathbf{k}^{\prime}}
\end{aligned}
$$

with

$$
f_{n \mathbf{k}}^{\prime}=\frac{1}{T} f^{\prime}\left(\frac{\varepsilon_{n \mathbf{k}}-\varepsilon_{F}}{T}\right)
$$

and where $\Sigma^{\prime}$ indicates that the term $m=n, \mathbf{k}^{\prime}=\mathbf{k}$ is omitted in the summation. Note that strictly speaking, these intermediary results are only valid for nondegenerate eigenvalues (otherwise $\delta \psi_{n \mathbf{k}}$ is not welldefined), but the end result is also valid for degenerate eigenvalues, see Levitt ${ }^{28}$ for an alternative derivation using contour integrals. We can determine $\delta \varepsilon_{F}$ by the condition that the total number of electrons is unchanged: $\sum_{\mathbf{k} \in \mathcal{B}_{\mathcal{N}}} \sum_{n=1}^{\infty} \delta f_{n \mathbf{k}}=0$ and therefore

$$
\delta \varepsilon_{F}=\frac{\left\langle D_{\text {loc }} \mid \delta V\right\rangle_{\Omega_{\mathcal{N}}}}{\mathcal{N} D}
$$

where we have introduced

$$
\begin{aligned}
D & =-\frac{1}{\mathcal{N}|\Omega|} \sum_{\mathbf{k} \in \mathcal{B}_{\mathcal{N}}} \sum_{n=1}^{\infty} f_{n \mathbf{k}}^{\prime} \\
D_{\mathrm{loc}}(\mathbf{r}) & =-\frac{1}{\mathcal{N}|\Omega|} \sum_{\mathbf{k} \in \mathcal{B}_{\mathcal{N}}} \sum_{n=1}^{\infty} f_{n \mathbf{k}}^{\prime}\left|u_{n \mathbf{k}}(\mathbf{r})\right|^{2}
\end{aligned}
$$


the total density of states per unit volume at the Fermi level, and the local density of states.

We can now obtain the integral kernel of $\chi_{0}$ both in real space and in reciprocal space. For the former, we simply identify using the formula $\delta \rho(\mathbf{r})=$ $\int_{\Omega_{\mathcal{N}}} \chi_{0}\left(\mathbf{r}, \mathbf{r}^{\prime}\right) \delta V\left(\mathbf{r}^{\prime}\right) \mathrm{d} \mathbf{r}^{\prime}$. For the latter, note that $\chi_{0}$ is invariant by the lattice translations $\mathcal{R}$, and can therefore be described by its Bloch matrix $\chi_{0}\left(\mathbf{q}, \mathbf{G}, \mathbf{G}^{\prime}\right)$, where $\mathbf{q} \in \mathcal{B}_{\mathcal{N}}, \mathbf{G}, \mathbf{G}^{\prime} \in \mathcal{R}^{*}$, describing the density response at wavelength $\mathbf{q}+\mathbf{G}$ to a total potential perturbation at wavelength $\mathbf{q}+\mathbf{G}^{\prime}$. Using the convention $\frac{f_{n \mathbf{k}}-f_{n \mathbf{k}}}{\varepsilon_{n \mathbf{k}}-\varepsilon_{n \mathbf{k}}}=f_{n \mathbf{k}}^{\prime}$, we obtain

$$
\begin{aligned}
\chi_{0}\left(\mathbf{r}, \mathbf{r}^{\prime}\right) & =\sum_{\mathbf{k}^{\prime} \in \mathcal{B}_{\mathcal{N}}} \sum_{\mathbf{k} \in \mathcal{B}_{\mathcal{N}}} \sum_{m=1}^{\infty} \sum_{n=1}^{\infty} \frac{f_{n \mathbf{k}}-f_{m \mathbf{k}^{\prime}}}{\varepsilon_{n \mathbf{k}}-\varepsilon_{m \mathbf{k}^{\prime}}} \psi_{n \mathbf{k}}^{*}(\mathbf{r}) \psi_{m \mathbf{k}^{\prime}}(\mathbf{r}) \psi_{n \mathbf{k}}\left(\mathbf{r}^{\prime}\right) \psi_{m \mathbf{k}^{\prime}}^{*}\left(\mathbf{r}^{\prime}\right)+\frac{|\Omega|}{\mathcal{N}} \frac{D_{\mathrm{loc}}(\mathbf{r}) D_{\mathrm{loc}}\left(\mathbf{r}^{\prime}\right)}{D} \\
\chi_{0}\left(\mathbf{q}, \mathbf{G}, \mathbf{G}^{\prime}\right) & =\frac{1}{\mathcal{N}|\Omega|} \sum_{\mathbf{k} \in \mathcal{B}_{\mathcal{N}}} \sum_{m=1}^{\infty} \sum_{n=1}^{\infty} \frac{f_{n \mathbf{k}}-f_{m, \mathbf{k}-\mathbf{q}}}{\varepsilon_{n \mathbf{k}}-\varepsilon_{m, \mathbf{k}-\mathbf{q}}}\left\langle u_{m, \mathbf{k}-\mathbf{q}} \mid e^{-i \mathbf{G} \cdot \mathbf{r}} u_{n \mathbf{k}}\right\rangle_{\Omega}\left\langle u_{n \mathbf{k}} \mid e^{i \mathbf{G}^{\prime} \cdot \mathbf{r}} u_{m, \mathbf{k}-\mathbf{q}}\right\rangle_{\Omega} \\
& +\delta_{\mathbf{q} \mathbf{0}} \frac{1}{D}\left\langle e^{i \mathbf{G} \cdot \mathbf{r}} \mid D_{\mathrm{loc}}\right\rangle_{\Omega}\left\langle D_{\mathrm{loc}} \mid e^{i \mathbf{G}^{\prime} \cdot \mathbf{r}}\right\rangle_{\Omega} .
\end{aligned}
$$

In both these expressions, the second term, arising from the variable Fermi level, imposes charge neutrality: $\int_{\Omega_{\mathcal{N}}} \delta \rho(\mathbf{r}) \mathrm{d} \mathbf{r}=0$ for all $\delta V$.

For $\mathbf{q} \neq \mathbf{0}$, we can take the thermodynamic limit $\mathcal{N} \rightarrow \infty$ and obtain the usual Adler-Wiser formula

$$
\begin{aligned}
& \lim _{\mathcal{N} \rightarrow \infty} \chi_{0}\left(\mathbf{q}, \mathbf{G}, \mathbf{G}^{\prime}\right) \\
& =\frac{1}{(2 \pi)^{3}} \int_{k \in \mathcal{B}} \sum_{n=1}^{\infty} \sum_{m=1}^{\infty} \frac{f_{n \mathbf{k}}-f_{m, \mathbf{k}-\mathbf{q}}}{\varepsilon_{n \mathbf{k}}-\varepsilon_{m, \mathbf{k}-\mathbf{q}}} A_{m n \mathbf{k}}\left(\mathbf{q}, \mathbf{G}, \mathbf{G}^{\prime}\right) \mathrm{d} \mathbf{k} .
\end{aligned}
$$

with matrix elements

$$
\begin{aligned}
& A_{m n \mathbf{k}}\left(\mathbf{q}, \mathbf{G}, \mathbf{G}^{\prime}\right) \\
& \quad=\left\langle u_{m, \mathbf{k}-\mathbf{q}} \mid e^{-i \mathbf{G} \cdot \mathbf{r}} u_{n \mathbf{k}}\right\rangle_{\Omega}\left\langle u_{n \mathbf{k}} \mid e^{i \mathbf{G}^{\prime} r} u_{m, \mathbf{k}-\mathbf{q}}\right\rangle_{\Omega} .
\end{aligned}
$$

Of particular interest is the long-wavelength limit $\mathbf{q} \rightarrow \mathbf{0}$, $\mathbf{G}=\mathbf{0}, \mathbf{G}^{\prime}=\mathbf{0}$, in which case $A_{m n \mathbf{k}}=\delta_{m n}$ and

$$
\begin{aligned}
\lim _{\mathbf{q} \rightarrow 0} \lim _{\mathcal{N} \rightarrow \infty} \chi_{0}(\mathbf{q}, \mathbf{0}, \mathbf{0}) & =\frac{1}{(2 \pi)^{3}} \int_{\mathcal{B}} \sum_{n=1}^{\infty} f_{n, \mathbf{k}}^{\prime} \mathrm{d} \mathbf{k} \\
& =-\lim _{\mathcal{N} \rightarrow \infty} D,
\end{aligned}
$$

the negative of the density of states at the Fermi level of this system. In the limit of zero temperature, $f_{n \cdot \mathbf{k}}^{\prime}$ localizes on the Fermi surface, and $D$ converges to a finite negative value if the system is conducting, and to zero if it is not. For insulating and semiconducting systems, expanding $u_{m, \mathbf{k}+\mathbf{q}}$ to first order (the " $\mathbf{k} \cdot \mathbf{p}$ perturbation theory"), we obtain that for $\mathbf{q}$ small, $\chi_{0}(\mathbf{q}, \mathbf{0}, \mathbf{0})$ is of the order of $|\mathbf{q}|^{2}$ and both $\chi_{0}(\mathbf{q}, \mathbf{G}, \mathbf{0})$ and $\chi_{0}\left(\mathbf{q}, \mathbf{G}^{\prime}, \mathbf{0}\right)$ are of order of $|\mathbf{q}|$ for $\mathbf{G}, \mathbf{G}^{\prime} \neq \mathbf{0}$.

This has strong consequences for the eigenvalues of the operator $\varepsilon^{\dagger}=1-\chi_{0} K$. Indeed, in the RPA,

$$
\varepsilon^{\dagger}\left(\mathbf{q}, \mathbf{G}, \mathbf{G}^{\prime}\right)=\delta_{\mathbf{G G}^{\prime}}-\chi_{0}\left(\mathbf{q}, \mathbf{G}, \mathbf{G}^{\prime}\right) \frac{4 \pi}{\left|\mathbf{q}+\mathbf{G}^{\prime}\right|^{2}}
$$

has the same eigenvalues as the symmetrized operator

$$
\begin{aligned}
& \left(1-v_{c}^{1 / 2} \chi_{0} v_{c}^{1 / 2}\right)\left(\mathbf{q}, \mathbf{G}, \mathbf{G}^{\prime}\right) \\
& \quad=\delta_{\mathbf{G G}^{\prime}}-4 \pi \frac{1}{|\mathbf{q}+\mathbf{G}|} \chi_{0}\left(\mathbf{q}, \mathbf{G}, \mathbf{G}^{\prime}\right) \frac{1}{\left|\mathbf{q}+\mathbf{G}^{\prime}\right|} .
\end{aligned}
$$

It follows from the considerations above that this operator diverges as $1 /|\mathbf{q}|^{2}$ for $\mathbf{q}$ small enough in the case of metals or systems at finite temperature, but not for insulators and semiconductors (see Cancès and Lewin ${ }^{29}$ as well as Levitt ${ }^{28}$ for a careful analysis). When computing for instance on a supercell $L \times 1 \times 1$ extended in one dimension, the lowest values of $\mathbf{q}$ allowed in $\mathcal{B}_{L}$ are of order $1 / L$. The result is that the condition number $\kappa$ of $\varepsilon^{\dagger}$ grows like $1 / L^{2}$ for metals: this is the source of charge sloshing. For insulators and semiconductors, no such divergence is present and the condition number is independent of the system size. In this case,

$$
\varepsilon_{r}=\lim _{\mathbf{q} \rightarrow \mathbf{0}} \frac{1}{\varepsilon^{-1}(\mathbf{q}, \mathbf{0}, \mathbf{0})}>1
$$

is the macroscopic dielectric constant (relative permittivity). Since the matrix element $\varepsilon^{-1}(\mathbf{q}, \mathbf{0}, \mathbf{0})$ is an upper bound on the lowest eigenvalue of $\varepsilon^{-1}$, it follows that $\kappa$ is at least higher than the dielectric constant. Therefore, $\kappa$ can be rather large for extended systems even if they are not conducting.

\section{B. Neglect of local field effects and dielectric functions}

Since bad convergence is linked to long-wavelength modes, it is useful to ignore lattice-scale details ("neglect of local field effects"). Under this approximation, the operator $\chi_{0}$ is modeled as a translation-invariant operator characterized by its Fourier multiplier $\chi_{0}(\mathbf{q})$. Under the 
RPA, the dielectric operator is also translation-invariant, with

$$
\varepsilon(\mathbf{q})=1-\frac{4 \pi \chi_{0}(\mathbf{q})}{|\mathbf{q}|^{2}}
$$

This relatively crude approximation is still able to reproduce at a qualitative level the difference between metals, semiconductors and insulators.

For metals, $\lim _{\mathbf{q} \rightarrow \mathbf{0}} \chi_{0}^{\text {metal }}(\mathbf{q})=-D$ for $\mathbf{q}$ small, where $D$ is the density of states per unit volume at the Fermi level. It follows that an appropriate approximation of $\varepsilon$ for $\mathbf{q}$ small is

$$
\varepsilon^{\text {metal }}(\mathbf{q})=\frac{4 \pi D+|\mathbf{q}|^{2}}{|\mathbf{q}|^{2}}
$$

Note that this result is the same as that obtained in the Thomas-Fermi theory of the electron gas. Indeed, in Thomas-Fermi theory, the potential-to-density mapping is given by $\rho=C_{\mathrm{TF}}\left(\varepsilon_{F}-V\right)_{+}^{3 / 2}$ where $C_{\mathrm{TF}}$ is a universal constant, and therefore

$$
\chi_{0}^{\mathrm{TF}}\left(\mathbf{r}, \mathbf{r}^{\prime}\right)=-\frac{3}{2} C_{\mathrm{TF}}^{\frac{2}{3}} \rho(r)^{\frac{1}{3}} \delta\left(\mathbf{r}-\mathbf{r}^{\prime}\right) .
$$

In the case of the homogeneous electron gas where $\rho$ is constant, we recover (39), with

$$
D^{\mathrm{TF}}=\frac{3}{2} C_{\mathrm{TF}}^{\frac{2}{3}} \rho^{\frac{1}{3}}
$$

The equation $(39)$ is also consistent with the small-q behavior of the dielectric function derived from the exact independent-particle susceptibility of the free electron gas (the Lindhard formula).

For insulators or semiconductors, as we have seen above, a more appropriate approximation of $\chi_{0}(\mathbf{q})$ for $\mathbf{q}$ small is $-\mathbf{q}^{T} \sigma_{0} \mathbf{q}$, where $\sigma_{0}$ is a unitless materialdependent symmetric positive definite matrix, with the physical meaning of a polarizability per unit volume. The dielectric function is then

$$
\varepsilon(\mathbf{q})=1+4 \pi \frac{\mathbf{q}^{T} \sigma_{0} \mathbf{q}}{|\mathbf{q}|^{2}}
$$

In the isotropic case where $\sigma_{0}$ is a scalar, $\varepsilon_{r}=\varepsilon(\mathbf{0})=$ $1+4 \pi \sigma_{0}$ is the dielectric constant of the material.

The concept of a translation-invariant dielectric function is only meaningful at long wavelengths (small q), where local field effects can be ignored. At larger $\mathbf{q}$ one can simply extrapolate to a reasonable function. In Figure 2 we plot approximate dielectric functions for three bulk materials: aluminium (Al), a metal, gallium arsenide (GaAs), a semiconductor, and silica $\left(\mathrm{SiO}_{2}\right)$, an insulator. For the metal, we used the formula (39) above, with $4 \pi D=1$. For the semiconductor and the insulator, we used the empirical formula

$$
\begin{aligned}
\chi_{0}^{\text {dielectric }}(\mathbf{q}) & =-\frac{\left(\varepsilon_{r}-1\right)|\mathbf{q}|^{2}}{4 \pi\left(1+\left(\varepsilon_{r}-1\right) \frac{|\mathbf{q}|^{2}}{k_{\mathrm{TF}}^{2}}\right)} \\
\varepsilon^{\text {dielectric }}(\mathbf{q}) & =\frac{\varepsilon_{r}+\left(\varepsilon_{r}-1\right) \frac{|\mathbf{q}|^{2}}{k_{\mathrm{TF}}^{2}}}{1+\left(\varepsilon_{r}-1\right) \frac{|\mathbf{q}|^{2}}{k_{\mathrm{TF}}^{2}}}
\end{aligned}
$$

This is a two-parameter simplification of the Resta mode $\sqrt{30}$ chosen to reproduce the correct behavior at zero and at infinity. We used for $\varepsilon_{r}$ the macroscopic isotropic electronic dielectric constants $\left(2.4\right.$ for $\mathrm{SiO}_{2}$ and 14 for GaAs), and set $k_{\mathrm{TF}}=1$.

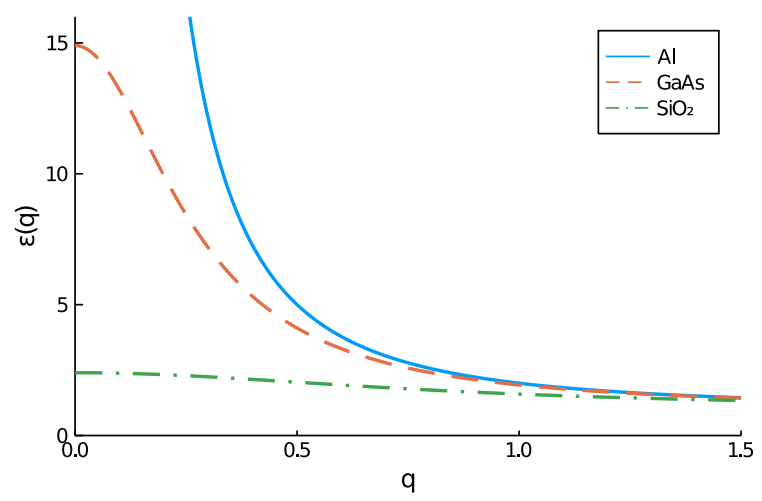

FIG. 2. Approximate dielectric functions of aluminium, gallium arsenide and silica.

\section{Preconditioners for homogeneous systems}

Several preconditioners have been proposed in the literature. The simplest class is that of homogeneous preconditioners, which simply act as multipliers in reciprocal space. The first such preconditioner is Kerker's scheme ${ }^{16}$, derived using Thomas-Fermi theory:

$$
P_{\text {Kerker }}^{-1}(\mathbf{q})=\frac{1}{\varepsilon^{\text {metal }}(\mathbf{q})}=\frac{|\mathbf{q}|^{2}}{k_{\mathrm{TF}}^{2}+|\mathbf{q}|^{2}},
$$

where $k_{\mathrm{TF}}$ is the Thomas-Fermi screening wavevector. This is exactly the metallic dielectric function (39), with $k_{\mathrm{TF}}=\sqrt{4 \pi D}$.

This preconditioner is appropriate for metals but not for insulators and semiconductors, and therefore the Kerker preconditioner has been modified to accommodate different types of dielectric behavior, for instance by the truncated Kerker scheme $P_{\text {trunc }}(\mathbf{q})=$ $P_{\text {Kerker }}\left(\max \left(|\mathbf{q}|, q_{\min }\right)\right)$ for some $q_{\min }$, or by using the Resta model as preconditioner ${ }^{3 / 18 \mid 31}$. We use a dielectric preconditioner based on the dielectric model prescribed in (43):

$$
P_{\text {dielectric }}^{-1}(\mathbf{q})=\frac{1}{\varepsilon^{\text {dielectric }}(\mathbf{q})}=\frac{1+\left(\varepsilon_{r}-1\right) \frac{|\mathbf{q}|^{2}}{k_{\mathrm{TF}}^{2}}}{\varepsilon_{r}+\left(\varepsilon_{r}-1\right) \frac{|\mathbf{q}|^{2}}{k_{\mathrm{TF}}^{2}}} .
$$

When a preconditioner is chosen, the condition number of the iterative scheme is equal to the ratio of largest to lowest eigenvalues of $P^{-1} \varepsilon^{\dagger}$. It follows that the condition number $\kappa$ of $P^{-1} \varepsilon^{\dagger}$ is small (and therefore convergence is rapid) when (a) $\varepsilon$ is well-approximated by a homogeneous dielectric function (and therefore the system is 
homogeneous enough), and (b) the dielectric function is well-approximated by the selected preconditioner (and therefore the parameters are selected appropriately). Selecting the wrong preconditioner risks damping too little or too much the long-wavelength modes, resulting in slow convergence.

\section{COMPUTATIONAL RESULTS ON HOMOGENEOUS SYSTEMS}

To compare different strategies of preconditioning numerically we will consider six testcases in the form of elongated supercells of silica $\left(\mathrm{SiO}_{2}\right)$, gallium arsenide (GaAs) and aluminium ( $\mathrm{Al})$ as simple examples of a bulk insulator, semiconductor and metal respectively.

To generate these test systems we repeated the conventional cubic (for GaAs and $\mathrm{Al}$ ) or trigonal (for $\mathrm{SiO}_{2}$ ) unit cells of the respective materials roughly 20 or 40 times along the (001) surface. All calculations have been performed in the densityfunctional toolkit (DFTK) ${ }^{32}$, in a plane-wave basis, using the Perdew-Burke-Ernzerhof (PBE) exchange-correlation functionals ${ }^{33}$ as implemented in the libxi ${ }^{34}$ library, and Godecker-Teter-Hutter pseudopotentials ${ }^{35}$. We used a kinetic energy cutoff of 20 Hartree and Monkhorst-Pack grids with a maximal spacing of $0.3 \mathrm{Bohr}^{-1}$. For aluminium a Gaussian smearing scheme with width of 0.001 Hartree was used. We chose tight convergence tolerances for the diagonalization during the SCF procedure to avoid any slowdown due to inaccurately represented bands. To avoid symmetry effects which would result in faster convergence not representative of larger-scale computations, we slightly perturbed the atomic positions and the initial guess density. We refer to our github repository of supporting information ${ }^{36}$ for full computational details, including instructions on how to reproduce all the results in this paper.

We show in Table $\mathbb{I}$ the results of the SCF procedure with Anderson acceleration (history size $m=10$ ) in four cases: without preconditioning $(P=1)$, with the dielectric preconditioner (45) (using the dielectric constants of $\mathrm{SiO}_{2}$ and $\mathrm{GaAs}$ ), and with the Kerker preconditioner. In all cases we took $k_{\mathrm{TF}}=1$. In order to avoid selecting good stepsizes manually, we estimated the lowest and largest eigenvalues $\lambda_{\min }$ and $\lambda_{\max }$ of $P^{-1} \varepsilon^{\dagger}$ at convergence, and used these to determine the optimal stepsize from (14). The eigenvalues were determined iteratively using the Arnoldi method. At each step, the action of $\varepsilon^{\dagger}=1-K \chi_{0}$ on a vector was computed by solving the Sternheimer equations. A similar method was used by Wilson and coworkers $37 \mid 38$ to approximate the dielectric operator. For technical reasons, we used the Teter reparametrization of the local-density approximation (LDA $)^{35}$ in the exchange-correlation kernel for the computation of $K$ instead of the PBE functional used in the SCF computations themselves.

From the eigenvalues we also computed the condition number $\kappa=\frac{\lambda_{\max }}{\lambda_{\min }}$. This gives a simple intrinsic measure of the quality of the preconditioner in the linear regime, free from the noise associated with the Anderson method (nonlinear effects, stepsize, history length, implementation details). Since the eigenvalues can be slow to converge, we only report approximate values. Using a crude bound based on the residuals obtained in our Arnoldi scheme we estimate the error in the values for $\kappa$ to be less than $13 \%$ over all cases considered in this work.

Both the number of iterations required to converge the SCF to an energy error of $10^{-10}$ as well as the condition number $\kappa$ are summarized in Table [ The corresponding plots are shown in Figure 3.

The combinations of systems and preconditioners for which there is no significant growth in the number of iterations and condition numbers are highlighted in orange color in Table I. As predicted from the theory, Kerker preconditioning leads to system size independence for the metal $\mathrm{Al}$, and no preconditioning or dielectric preconditioning for the insulator $\mathrm{SiO}_{2}$ or the semiconductor GaAs. In the other cases, the condition number grows quadratically with the system size. On semiconductors, even if the unpreconditioned condition number is independent on system size, it can still be rather large (on the order of the dielectric constant) and therefore benefit from appropriate preconditioning.

On the diagonal in Table I, using a preconditioner adapted to the system at hand leads to a condition number between 2 and 4, and convergence in less than 20 iterations. Using the wrong preconditioner leads to degraded convergence. On $\mathrm{SiO}_{2}$ and $\mathrm{Al}$, convergence is eventually achieved even for inadequate preconditioners, albeit slowly. On GaAs however, the interference of strong nonlinear effects on the Anderson scheme leads to a quick divergence (see discussion in Section III).

The long-wavelength charge sloshing is responsible for slow convergence, as illustrated Figure 4 on the largest eigenmodes of $\varepsilon^{\dagger}$ in the case of unpreconditioned Al. Being eigenmodes of the almost periodic operator $\varepsilon^{\dagger}$, these modes are close to Bloch waves $e^{i \mathbf{q} \cdot \mathbf{r}} u(\mathbf{r})$ with $\mathbf{q}$ consistent with periodic boundary conditions on the computational domain and $u$ lattice-periodic. The largest eigenvalues are associated with small $\mathbf{q}$.

\section{PRECONDITIONERS FOR HETEROGENEOUS SYSTEMS}

As we have seen, for simple homogeneous systems a translation-invariant approximation of $\chi_{0}$ and therefore $\varepsilon^{-1}$ is appropriate. For more complex systems (for instance, periodic materials with large unit cells, clusters and surfaces), these are very often unsuccessful in significantly reducing the number of iterations, and several alternative preconditioners have been proposed.

The scheme in Raczkowski et al.17 employs a modified Thomas-Fermi-von Weizsäcker (TFW) model to derive an inexpensive approximation to the dielectric opera- 


\begin{tabular}{|c|c|c|c|c|c|c|c|c|c|}
\hline & \multirow[b]{2}{*}{$\mathcal{N}$} & \multicolumn{2}{|c|}{ None } & \multicolumn{2}{|c|}{$\begin{array}{c}\text { Dielectric } \\
\left(\mathrm{SiO}_{2}\right)\end{array}$} & \multicolumn{2}{|c|}{$\begin{array}{c}\text { Dielectric } \\
\text { (GaAs) }\end{array}$} & \multicolumn{2}{|c|}{ Kerker } \\
\hline & & it & $\kappa$ & it & $\kappa$ & it & $\kappa$ & it & $\kappa$ \\
\hline \multirow[t]{2}{*}{$\mathrm{SiO}_{2}$} & 21 & 13 & 3.2 & 13 & 3.5 & 22 & 10.2 & 33 & 103.1 \\
\hline & 39 & 12 & 3.2 & 16 & 3.5 & 22 & 10.7 & n.c. & 351.6 \\
\hline \multirow[t]{2}{*}{$\mathrm{GaAs}^{\mathrm{a}}$} & 20 & n.c. & 14.7 & n.c. & 9.3 & 10 & 2.4 & 23 & 31.2 \\
\hline & 40 & n.c. & 18.0 & n.c. & 11.3 & 10 & 2.7 & 38 & 100.9 \\
\hline \multirow[t]{2}{*}{$\mathrm{Al}$} & 20 & 29 & 58.1 & 22 & 30.5 & 15 & 7.2 & 10 & 3.9 \\
\hline & 40 & n.c. & 261.6 & n.c. & 141.3 & 24 & 27.8 & 10 & 2.5 \\
\hline
\end{tabular}

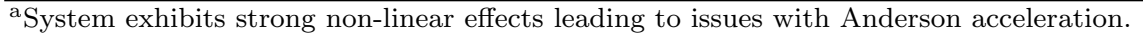

TABLE I. Number of iterations and condition number $\kappa$ for several homogeneous materials and preconditioners. $\mathcal{N}$ denotes the number of repeats of the conventional unit cell and "n.c." indicates that the SCF has not converged after 50 iterations. The cases where $\kappa$ increases by less than a factor of two when the system size is doubled are colored.

tor. However, the TFW model, like the simpler ThomasFermi model in (40), predicts metallic-like full screening (see Cancès and Ehrlacher ${ }^{39}$ for a detailed analysis), and is therefore unable to account for the difference in dielectric behavior between insulators, semiconductors and metals. In practice, we observed the convergence with this preconditioner to strongly degrade with system size for semiconductors and (especially) insulators, exactly as the Kerker scheme. A slightly generalized version of this approach has been proposed by Hasnip and Probert ${ }^{20}$, where the Kohn-Sham problem is dynamically mapped to a related auxiliary problem, which is solved self-consistently at each iteration in order to determine the preconditioned density for the next iteration. The "extrapolar method" of Anglade and Gonze $\mathrm{e}^{22}$, building on the earlier HIJ method ${ }^{21}$, is based on a full computation and inversion of the dielectric operator on a reduced system. It performs well, but is very costly, scaling like the fourth power of the number of electrons. The "elliptic preconditioner" of Lin and Yang ${ }^{[19}$ uses a model elliptic equation with variable coefficients as a dielectric model, approximating $\varepsilon^{-1}$ by the solution of the equation

$$
[\nabla \cdot(a(\mathbf{r}) \nabla)+4 \pi b(\mathbf{r})]\left(\varepsilon^{-1} V\right)=-\nabla^{2} V .
$$

In the case of constant coefficients $a$ and $b$, this method is able to reproduce the dielectric behavior of metals $(39)$ by taking $a=1, b=D$ and of insulators and semiconductors (42) by taking $b=0$ and $a$ equal to the dielectric constant. By manually constructing appropriate switching functions for $a$ and $b$, they were able to demonstrate good performance on hybrid systems consisting of a metallic and a vacuum region. However, this method requires manual tuning for the system at hand, in a fashion that is hard to automatize.

In this work, we propose to build an inhomogeneous preconditioner by approximating the independentparticle susceptibility $\chi_{0}$ instead of the dielectric operator $\varepsilon$. Consider an arbitrary finite system (not necessarily periodic) with orthonormal eigenfunctions $\varphi_{i}$, eigenvalues $\varepsilon_{i}$, Fermi level $\varepsilon_{F}$, occupations $f_{i}=f\left(\frac{\varepsilon_{i}-\varepsilon_{F}}{T}\right)$ and temperature $T$. Then, following the computations in Section IVA, $\chi_{0}$ can be written as

$$
\begin{aligned}
\chi_{0}\left(\mathbf{r}, \mathbf{r}^{\prime}\right) & =\sum_{i=1}^{\infty} \sum_{j=1}^{\infty} \frac{f_{i}-f_{j}}{\varepsilon_{i}-\varepsilon_{j}} \varphi_{i}^{*}(\mathbf{r}) \varphi_{j}(\mathbf{r}) \varphi_{i}\left(\mathbf{r}^{\prime}\right) \varphi_{j}^{*}\left(\mathbf{r}^{\prime}\right) \\
& +\frac{D_{\mathrm{loc}}(\mathbf{r}) D_{\mathrm{loc}}\left(\mathbf{r}^{\prime}\right)}{D}
\end{aligned}
$$

where

$$
\begin{gathered}
D=-\sum_{i=1}^{\infty} f_{i}^{\prime} \\
D_{\mathrm{loc}}(\mathbf{r})=-\sum_{i=1}^{\infty} f_{i}^{\prime}\left|\varphi_{i}\right|^{2}(\mathbf{r})
\end{gathered}
$$

are the density of states (not per volume, in contrast to the computations in $\overline{\mathrm{IV} \mathrm{A}}$ and localized density of states at the Fermi level.

The second term $\chi_{0}^{(2)}$ in 47 relates to the global variation of the Fermi level, is fully nonlocal and easily computable. The first term $\chi_{0}^{(1)}$ with its double sum over all states is where the complexity of building preconditioners lies. However, if we are interested only in describing long-range modes, we can write

$$
\begin{aligned}
\int \chi_{0}^{(1)}\left(\mathbf{r}, \mathbf{r}^{\prime}\right) V\left(\mathbf{r}^{\prime}\right) \mathrm{d} \mathbf{r}^{\prime} & \approx V(\mathbf{r}) \int \chi_{0}^{(1)}\left(\mathbf{r}, \mathbf{r}^{\prime}\right) \mathrm{d} \mathbf{r}^{\prime} \\
& =V(\mathbf{r}) \sum_{i=1}^{\infty} \sum_{j=1}^{\infty} \frac{f_{i}-f_{j}}{\varepsilon_{i}-\varepsilon_{j}} \varphi_{i}^{*}(\mathbf{r}) \varphi_{j}(\mathbf{r}) \delta_{i j} \\
& =-D_{\mathrm{loc}}(\mathbf{r}) V(\mathbf{r})
\end{aligned}
$$

where we have assumed that $\mathbf{r}^{\prime} \mapsto \chi_{0}^{(1)}\left(\mathbf{r}, \mathbf{r}^{\prime}\right)$ is localized around $\mathbf{r}$ at a scale smaller than the characteristic scale of variation of $V$. This process corresponds to condensing all of $\chi_{0}^{(1)}\left(\mathbf{r}, \mathbf{r}^{\prime}\right)$ on its diagonal (similar to the "mass lumping" used in the finite element community). 

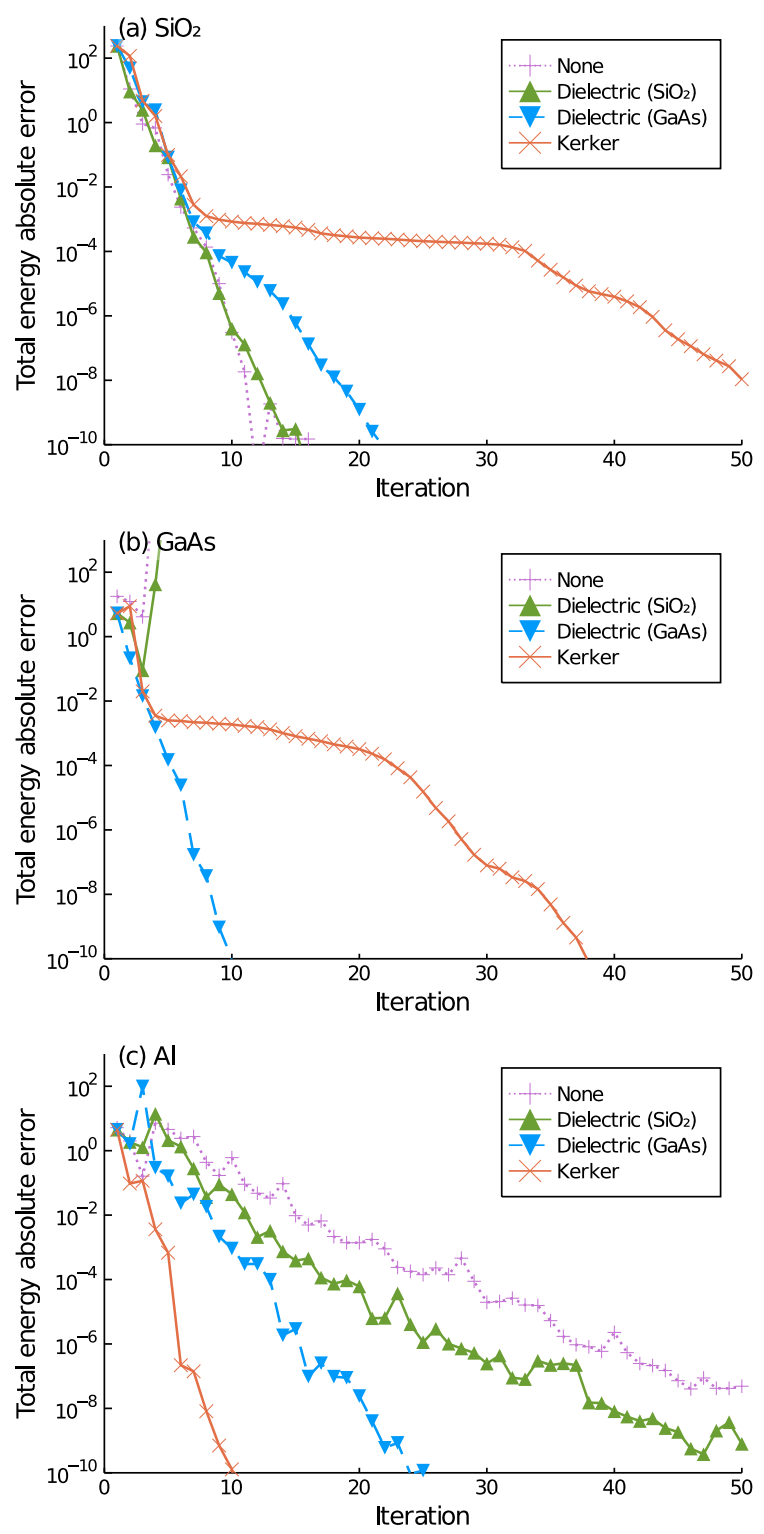

FIG. 3. Convergence plots for bulk systems. From top to bottom the plots show (a) 39 repeats of silica, (b) 40 repeats of gallium arsenide and (c) 40 repeats of aluminium. For GaAs the iterations for no preconditioner and for the dielectric $\left(\mathrm{SiO}_{2}\right)$ preconditioner start to diverge after 3 iterations.

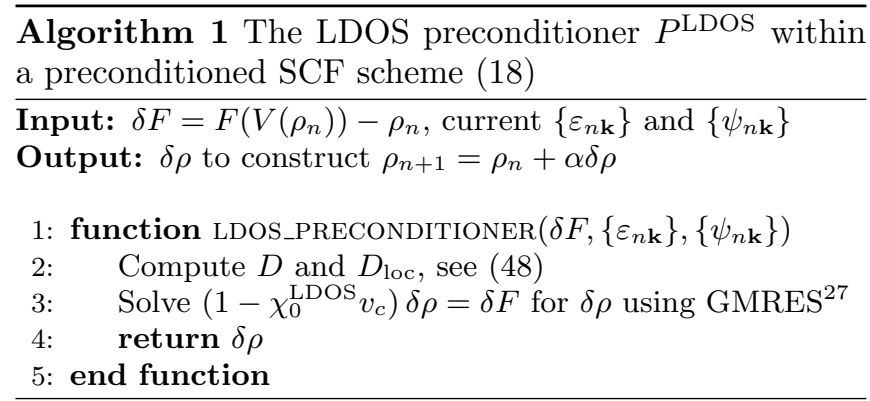

Remarkably, this large-scale averaging process involves

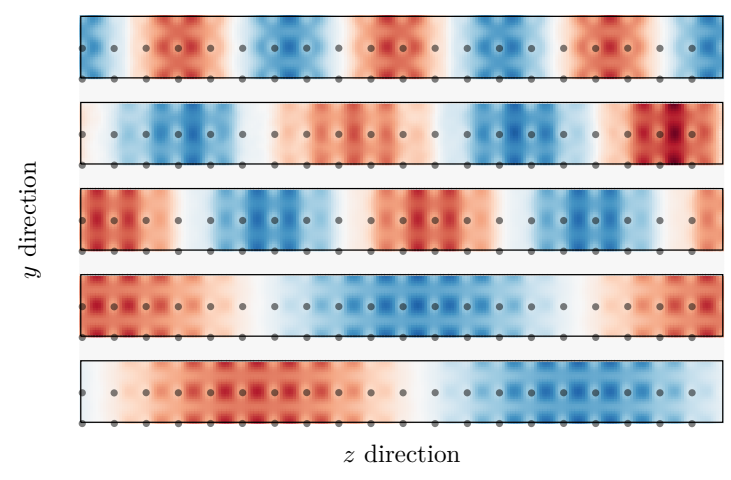

FIG. 4. The five most dominant eigenmodes of the dielectric adjoint operator $\varepsilon^{\dagger}$ for bulk aluminium with 40 repeats. The modes are plotted in real space, averaged over the $x$ direction, corresponding approximately (from top to bottom) to eigenvalues 24, 46, 47, 261 and 261. The modes display typical charge-sloshing behavior, moving electron density between extremal parts of the cell.

only the "diagonal" excitations $i=j$ in $\chi_{0}^{(1)}$, resulting in a tremendous reduction in complexity.

The final expression (and the central result of this paper) is

$$
\chi_{0}^{\mathrm{LDOS}}\left(\mathbf{r}, \mathbf{r}^{\prime}\right)=-D_{\mathrm{loc}}(\mathbf{r}) \delta\left(\mathbf{r}-\mathbf{r}^{\prime}\right)+\frac{D_{\mathrm{loc}}(\mathbf{r}) D_{\mathrm{loc}}\left(\mathbf{r}^{\prime}\right)}{D} .
$$

We use this to build a preconditioner

$$
P^{\mathrm{LDOS}}=\left(1-\chi_{0}^{\mathrm{LDOS}} v_{c}\right) .
$$

In our tests, including the exchange-correlation kernel $K_{\mathrm{XC}}$ in the preconditioner did not improve it, and therefore we stay within the RPA.

To apply $P^{-1}$ to a vector, we solve the linear equation iteratively using the GMRES method ${ }^{27}$, see Algorithm 1 . We first compute the local density of states, which is of the same complexity as computing the density. At each step of the iterative solver (line 3 of Algorithm 1), we have to apply $v_{c}$ and $\chi_{0}^{\mathrm{LDOS}}$ to a test vector. These are inexpensive operations since they scale linearly with the number of atoms. More precisely, applying $\chi_{0}^{\mathrm{LDOS}}$ only requires multiplications in real space and the Coulomb operator is applied as usual (using Fourier transforms and a multiplication in Fourier space). Both operations are needed for other steps in DFT computations, and therefore this method can readily be implemented in any DFT code, independently of the basis set used.

This preconditioner is completely parameter-free, and can be seen as a localized version of Kerker's preconditioning, to which it reduces when $D_{\text {loc }}$ is constant. By using the density of states of the system, it automatically adjusts the constant $k_{\mathrm{TF}}^{2}$ appearing in the Kerker preconditioner using the properties of the system under consideration. This systematizes the findings in Zhou et al. $\frac{18}{11}$ where this constant was adapted to the number of metallic atoms in a hybrid Au-MoS2 system. In 
contrast to the homogeneous preconditioners discussed in Section IV C, the LDOS-based approach furthermore does not assume that local field effects can be neglected, since the inverse of $P$ is computed iteratively, which is more appropriate to heterogenous systems.

Since the LDOS is localized on metallic regions, we can expect the approximation (50) to be accurate on systems made of simple, free electron-like metals and vacuum, and we will see in Section VII that this is indeed the case. On regions containing insulators and semiconductors, $D_{\text {loc }}(\mathbf{r})$ will be zero, such that (50) also performs well for almost inert materials like $\mathrm{SiO}_{2}$, where $\chi_{0} \approx 0$ is an appropriate approximation. This is in contrast to the preconditioner of Raczkowski et al. $\frac{17}{17}$ based on ThomasFermi theory, which incorrectly predicts a non-zero local density of states proportional to the cube root of the density (see (41)), and therefore in particular overscreens insulators. However, on semiconductors like gallium arsenide, our approximation corresponds to not preconditioning at all, which we saw in Section V yields a rather large condition number of the order of its dielectric constant. This can be traced to the fact that the approximation 49 completely neglects any type of polarizability and only considers the bulk reaction of free electrons.

To account for this and obtain a model which is suitable for semiconductors as well, we can simply add the susceptibility model of 43 :

$$
\chi_{0}^{\mathrm{LDOS}+\text { dielectric }}=\chi_{0}^{\mathrm{LDOS}}+\chi_{0}^{\text {dielectric }} .
$$

The rationale behind this empirical modification is that adding the dielectric model corrects for $\chi_{0}^{\mathrm{LDOS}}$ being zero in semiconducting regions while at the same time not having a large effect in metallic regions, where the local density of states term of $\chi_{0}^{\mathrm{LDOS}}$ is anyway dominating. Compared to the pure $\chi_{0}^{\mathrm{LDOS}}$ model, which is completely free of empirical parameters, this "LDOS+dielectric" preconditioner introduces the two free parameters $k_{\mathrm{TF}}$ and $\varepsilon_{r}$ from $\chi_{0}^{\text {dielectric }}$. As we will see in the following this does, however, allow the resulting LDOS+dielectric preconditioner to be much more effective on mixed systems containing semiconductors. Naturally further improvements are possible, at the expense of introducing additional empirical parameters. One aspect we have considered is the use of a switching function $L(\mathbf{r})$ going from 0 to 1 inside the semiconducting region of the system. This effectively activates $\chi_{0}^{\text {dielectric }}$ only in the semiconducting region, resulting in the "localized" preconditioner

$$
\begin{aligned}
\chi_{0}^{\text {localized }}\left(\mathbf{r}, \mathbf{r}^{\prime}\right) & =\chi_{0}^{\mathrm{LDOS}}\left(\mathbf{r}, \mathbf{r}^{\prime}\right) \\
& +\sqrt{L(\mathbf{r})} \chi_{0}^{\text {dielectric }}\left(\mathbf{r}, \mathbf{r}^{\prime}\right) \sqrt{L\left(\mathbf{r}^{\prime}\right)} .
\end{aligned}
$$

\section{COMPUTATIONAL RESULTS ON HETEROGENEOUS SYSTEMS}

In this section we consider a number of interfaces involving a combination of the materials we used previously
$\left(\mathrm{SiO}_{2}, \mathrm{GaAs}\right.$ and $\left.\mathrm{Al}\right)$ as well as vacuum. The computational details are the same as in Section V, with the exception of the $k$-point mesh, for which a maximal spacing of $0.15 \mathrm{Bohr}^{-1}$ was used, and the initial guess and structures, which were not randomized.

We built the surface systems by stacking $\mathcal{N}$ monolayers of the first material with $\mathcal{N}$ monolayers of the second material and, for $\mathrm{Al}+\mathrm{GaAs}+\mathrm{SiO}_{2}$, additionally $\mathcal{N}$ monolayers of the third material. For $\mathrm{SiO}_{2}$ and $\mathrm{GaAs}$ the (110) surface was employed, while the (100) surface was used for $\mathrm{Al}$. For cases involving vacuum the vacuum region was taken of the same width as the material layer. To avoid the emergence of surface states, which are highly localized and thus form another source of instability not of focus here, all silica surfaces were passivated with hydrogen. To make the lattices compatible, $\mathrm{Al}$ and $\mathrm{SiO}_{2}$ were compressed or expanded in the appropriate direction to adapt to GaAs. Note that we did not deform GaAs, where the band gap is most susceptible to strain. We tested the following preconditioners:

- no preconditioner $(P=1)$;

- the dielectric preconditioner 43 , with $\varepsilon_{r}=14$, $k_{\mathrm{TF}}=1$;

- the Kerker preconditioner 44 , with $k_{\mathrm{TF}}=1$;

- the parameter-free LDOS preconditioner based on 50);

- the LDOS+dielectric preconditioner based on 52 , with $\varepsilon_{r}=14, k_{\mathrm{TF}}=1$;

- on selected systems, the localized preconditioner based on 53), with the same parameters as the LDOS+dielectric preconditioner and $L(\mathbf{r})$ constructed as a linear combination of error functions chosen to be 1 inside the GaAs region and 0 outside;

- on selected systems, the TFW preconditioner of Raczkowski et al.17, using the implementation in Quantum ESPRESSO 4014 with the same parameters as for the other computations as far as possible. For these computations we use Quantum ESPRESSO's default damping value of 0.7 , but found results to be similar for other choices of damping. In contrast to the DFTK-computed curves, where the absolute error in the total energy is used directly to estimate the SCF error at each iteration, the Quantum ESPRESSO curves show the estimated scf accuracy quoted in the Quantum ESPRESSO output (which is the $v_{c}$-norm of the density difference squared).

As previously, we show the number of iterations to reach an energy error of $10^{-10}$ and the condition numbers $\kappa$ in Table II Cases where $\kappa$ increases by less than a factor of two as the system size is doubled are again highlighted in orange. In every system except for $\mathrm{GaAs}+\mathrm{SiO}_{2}$, the SCF procedures converge or are on the way to very slow 


\begin{tabular}{|c|c|c|c|c|c|c|c|c|c|c|c|c|c|}
\hline & \multirow[b]{2}{*}{$\mathcal{N}$} & \multicolumn{2}{|c|}{ None } & \multicolumn{2}{|c|}{ Dielectric } & \multicolumn{2}{|c|}{ Kerker } & \multicolumn{2}{|c|}{ LDOS } & \multicolumn{2}{|c|}{$\begin{array}{c}\text { LDOS+ } \\
\text { Dielectric }\end{array}$} & \multicolumn{2}{|c|}{ Localized } \\
\hline & & it & $\kappa$ & it & $\kappa$ & it & $\kappa$ & it & $\kappa$ & it & $\kappa$ & it & $\kappa$ \\
\hline \multirow[t]{2}{*}{$\mathrm{SiO}_{2}+$ vacuum } & 10 & 11 & 3.3 & 26 & 19.7 & 50 & 95.7 & 11 & 3.3 & 26 & 19.7 & & \\
\hline & 20 & 12 & 3.4 & 30 & 24.4 & n.c. & 351.5 & 12 & 3.4 & 30 & 21.7 & & \\
\hline \multirow[t]{2}{*}{ GaAs+vacuum } & 10 & 17 & 13.4 & 18 & 6.2 & 23 & 67.0 & 17 & 12.4 & 18 & 10.4 & 11 & 3.5 \\
\hline & 20 & 20 & 15.5 & 22 & 12.9 & n.c. & 312.2 & 20 & 15.5 & 22 & 12.9 & 13 & 4.6 \\
\hline \multirow{2}{*}{$\mathrm{Al}+$ vacuum } & 10 & 19 & 51.5 & 24 & 44.3 & 22 & 64.4 & 9 & 3.7 & 16 & 10.3 & & \\
\hline & 20 & 47 & 170.8 & 49 & 168.5 & n.c. & 323.9 & 9 & 3.5 & 20 & 10.5 & & \\
\hline \multirow[t]{2}{*}{$\mathrm{GaAs}+\mathrm{SiO}_{2}{ }^{\mathrm{a}}$} & 10 & 45 & 13.7 & 19 & 8.9 & 34 & 52.4 & 45 & 13.4 & 19 & 8.8 & 16 & 6.1 \\
\hline & 20 & n.c. & 18.2 & 20 & 10.2 & n.c. & 170.1 & n.c. & 18.2 & 20 & 10.2 & 24 & 10.3 \\
\hline \multirow{2}{*}{$\mathrm{Al}+\mathrm{SiO}_{2}$} & 10 & 43 & 93.1 & 29 & 33.6 & 30 & 50.9 & 17 & 6.1 & 20 & 9.2 & & \\
\hline & 20 & n.c. & 316.6 & n.c. & 118.4 & n.c. & 159.4 & 14 & 5.4 & 20 & 10.1 & & \\
\hline \multirow[t]{2}{*}{$\mathrm{Al}+\mathrm{GaAs}$} & 10 & n.c. & 144.0 & 24 & 22.4 & 16 & 9.0 & 15 & 7.2 & 11 & 3.5 & & \\
\hline & 20 & n.c. & 485.0 & 40 & 59.0 & 26 & 28.8 & 26 & 21.4 & 13 & 5.0 & & \\
\hline $\mathrm{Al}+\mathrm{GaAs}+\mathrm{SiO}_{2}$ & 10 & n.c. & 149.5 & 34 & 50.4 & 36 & 62.9 & 26 & 21.5 & 19 & 9.0 & 21 & 10.4 \\
\hline
\end{tabular}

TABLE II. Number of iterations and condition number $\kappa$ for several heterogeneous materials and preconditioners. $\mathcal{N}$ denotes the number of repeats of the conventional unit cell and "n.c." indicates that the SCF has not converged after 50 iterations. The cases where $\kappa$ increases by less than a factor of two when the system size is doubled are colored.

convergence when they are stopped after 50 iterations. In $\mathrm{GaAs}+\mathrm{SiO}_{2}$ however, as in bulk GaAs, the interference of strong nonlinear effects on the Anderson scheme leads to a quick divergence for some preconditioners.

None of the homogeneous strategies are able to perform well on all surfaces. This is particularly clear in the case of systems containing aluminium: for instance, on the large $\mathrm{Al}+\mathrm{SiO}_{2}$ and $\mathrm{Al}+$ vacuum test case, all have condition numbers in the hundreds, with the SCF converging very slowly (in around 50 iterations or more). Even on nonmetallic systems, the homogeneous preconditioners are not able to achieve condition numbers less than 10 on the large testcases, with the exception of the unpreconditioned $\mathrm{SiO}_{2}$ surface.

By contrast, our parameter-free LDOS-based scheme performs fairly well, achieving condition numbers less than 10 for all systems not containing the semiconductor GaAs, with associated quick convergence of the SCF. Even for the systems containing GaAs, condition numbers are reasonable, never exceeding 25 . The convergence is accordingly relatively quick, with the exception of the $\mathrm{GaAs}+\mathrm{SiO}_{2}$ system mentioned above.

On systems containing GaAs, we tested the LDOS+dielectric and the localized preconditioner. In $\mathrm{Al}+\mathrm{GaAs}$, adding the dielectric susceptibility model results in a low condition number, suggesting that it does not significantly hamper the good model in the metallic region. In the GaAs surface (where the LDOS is constant zero), plainly adding the susceptibility model does not significantly improve things. However, localizing it on the GaAs results in a low condition number. The improvement is not as marked on the $\mathrm{GaAs}+\mathrm{SiO}_{2}$, where surface effects might play a larger role. Finally, the larger $\mathrm{Al}+\mathrm{GaAs}+\mathrm{SiO}_{2}$ is adequately preconditioned with the LDOS+dielectric model, without need of localizing it.

In Figure 5, we compare our preconditioner against
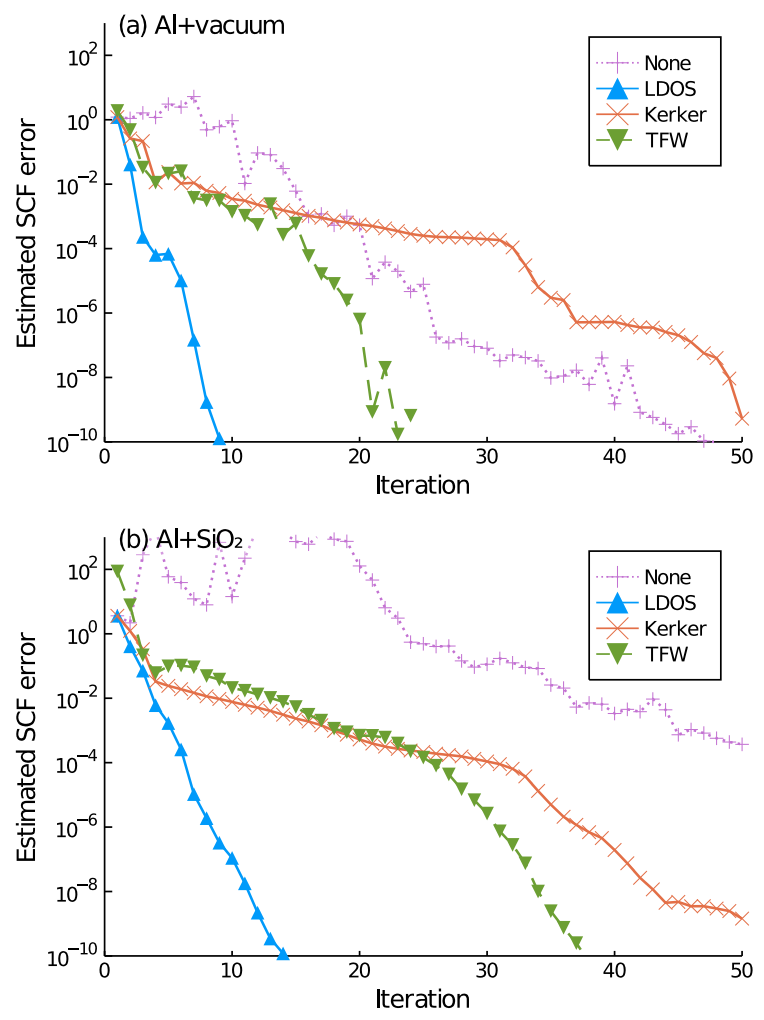

FIG. 5. Convergence plots for (a) the Al+vacuum and (b) the $\mathrm{Al}+\mathrm{SiO}_{2}$ systems with 20 repeats.

the inhomogeneous TFW preconditioner of Raczkowski et al. $\frac{17}{17}$ on two specific metallic systems, for which the TFW model should be adequate in the metallic region: the $\mathrm{Al}$ surface, and the $\mathrm{Al}+\mathrm{SiO}_{2}$ interface. On both these systems, our LDOS preconditioner converges in about 10 iterations. By contrast, the TFW preconditioner is less 
efficient, the effect being especially pronounced in the $\mathrm{Al}+\mathrm{SiO}_{2}$ system where the TFW model is very inadequate in the $\mathrm{SiO}_{2}$ region, being based on the density instead of the LDOS for determining dielectric properties. We illustrate this point by plotting in Figure 6 the pseudodensity and the LDOS in these two systems. As is apparent, the density is drastically different in the vacuum and the $\mathrm{SiO}_{2}$ regions, although both have very similar screening properties, having no free electrons. By contrast, the LDOS is able to correctly recognize the lack of free electrons in the insulator region.

In Figure 6, we also plot the LDOS computed at two different temperatures: that used in the SCF, and a higher temperature. The LDOS at the SCF temperature is very noisy because of the insufficient Brillouin zone sampling relative to the small temperature used. In contrast the LDOS at the elevated temperature is more regular, closer to the actual LDOS of this system. Nevertheless, we found the LDOS preconditioning to be relatively insensitive to the noise at the SCF temperature in our test cases. Should this manifest as an issue in other calculations, a higher smearing temperature could be used for determining the LDOS, or the LDOS could be artificially smoothed.

Apart from the rather artificial (but illustrative) test systems considered here, we have also applied the LDOS preconditioner to further tests taken from the scf-xn test suite 23 . These similarly demonstrate that this preconditioner, with its ability to switch on and off according to the character of the system (metallic or not), is especially applicable to mixed systems containing metals, insulators and vacuum. For example, using a kinetic energy cutoff of 35 Hartree and otherwise the computational parameters from Woods et al. ${ }^{[23}$, we found that the LDOS preconditioner, gives a rapid and steady convergence to an energy error of $10^{-8}$ in around 10 iterations for a variety of test cases such as a caffeine molecule, a slab of gold $\left(\mathrm{Au}_{40}\right.$ supercell), or a cluster of 51 water molecules, and is therefore more appropriate as a "black-box" preconditioner than the homogeneous schemes. It was however as ineffective as homogeneous schemes in "complicated" inhomogeneous metals with localized orbitals close to the Fermi level.

\section{CONCLUSION}

A novel preconditioning scheme for the self-consistent Kohn-Sham equations based on the local density of states (LDOS) has been constructed and its performance evaluated in representative test systems. We showed our scheme to be broadly applicable for large inhomogeneous systems involving metals, vacuum and insulators. The key idea of the proposed preconditioning scheme is to approximate the independent-particle susceptibility $\chi_{0}$ by its large-scale component $(50)$, which only involves the readily computable LDOS. The result is a preconditioner which is simple to implement, inexpensive to com- (a) Al+vacuum

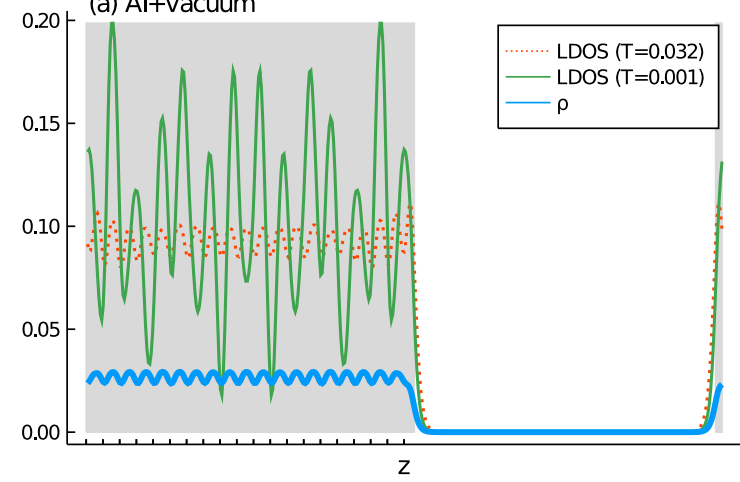

(b) $\mathrm{Al}+\mathrm{SiO}_{2}$

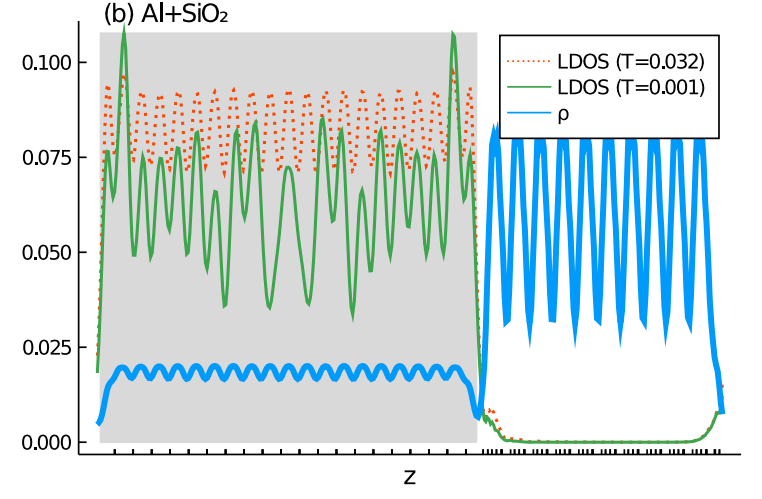

FIG. 6. Local density of states (LDOS) and pseudodensity $\rho$ (thick line) of the (a) $\mathrm{Al}+$ vacuum system and the (b) $\mathrm{Al}+\mathrm{SiO}_{2}$ system. Values are averaged in the $x y$-plane. The LDOS is shown both for the temperature used for preconditioning and during the SCF ( $T=0.001$ Hartree) and at a higher temperature, where it is more accurate due to insufficient Brillouin zone sampling. The part of the cell occupied by aluminium is shown in grey background and atomic positions projected onto the $z$-axis are shown with a tick.

pute and free of any parameters. By nature of the LDOS the scheme automatically adapts to the local environment in mixed systems, effectively interpolating between a Kerker-like treatment in metallic regions and no preconditioning where the local density of states is zero.

We believe our proposed LDOS-based preconditioning scheme to be an important step towards a generally applicable, parameter-free and black-box preconditioner for SCF iterations. In its present state there are, however, a number of limitations. Firstly, as we have discussed the LDOS is not able to distinguish insulators and semiconductors. As a remedy we have investigated an empirical correction by adding a dielectric model parametrized in the macroscopic dielectric constant. The resulting preconditioner performs very well in cases with semiconducting regions, but an appropriate dielectric constant has to be selected.

In order to go beyond the current approach, one could try to improve on the diagonal approximation for $\chi_{0}$ to represent polarizability effects. Unlike the LDOS, the polarizability is however more expensive to compute and 
cannot be easily localized $^{42}$, making it difficult to develop a fully transferable preconditioning scheme. Another limitation of our current preconditioner is that it only focuses on preventing long-range charge-sloshing and none of the other sources of ill-conditioning in SCF schemes (electronic phase transitions and localized states close to the Fermi level). We intend to tackle this in future work. We believe the idea of approximating $\chi_{0}$ instead of directly tackling the inverse dielectric operator to be fruitful for this endeavor as well, because $\chi_{0}$ is susceptible to additive approximations (unlike $\varepsilon^{-1}$ ). Therefore different physics (charge transfer, polarizability...) can be summed up, resulting in a composable dielectric model. Finally, we mention that inexpensive approximations of the dielectric operator can be useful in other contexts as well, such as for preconditioning geometry optimization, in response computations, or for constructing screened Coulomb interactions.

\section{ACKNOWLEDGMENTS}

This project has received funding from the ISCD (Sorbonne Université) and from the European Research Council (ERC) under the European Union's Horizon 2020 research and innovation program (grant agreement No 810367). We gratefully acknowledge stimulation discussions with E. Cancès, X. Gonze, P. Hasnip, L. Lin, and C. Yang.
* michael.herbst@inria.fr

$\dagger$ antoine.levitt@inria.fr

1 P. Hohenberg and W. Kohn, Physical Review 136, B864 (1964)

${ }^{2}$ W. Kohn and L. J. Sham, Physical Review 140, A1133 (1965)

${ }^{3}$ G. Kresse and J. Furthmüller, Physical Review B 54, 11169 (1996)

4 J. K. Norskov, F. Abild-Pedersen, F. Studt, and T. Bligaard, Proceedings of the National Academy of Sciences 108, 937 (2011)

${ }^{5}$ P. J. Hasnip, K. Refson, M. I. J. Probert, J. R. Yates, S. J. Clark, and C. J. Pickard, Philosophical Transactions of the Royal Society A: Mathematical, Physical and Engineering Sciences 372, 20130270 (2014)

${ }^{6}$ D. Morgan, G. Ceder, and S. Curtarolo, Measurement Science and Technology 16, 296 (2005)

A. Jain, G. Hautier, C. J. Moore, S. Ping Ong, C. C. Fischer, T. Mueller, K. A. Persson, and G. Ceder, Computational Materials Science 50, 2295 (2011)

W. Setyawan, R. M. Gaume, S. Lam, R. S. Feigelson, and S. Curtarolo, ACS Combinatorial Science 13, 382 (2011).

9 J. Greeley, T. F. Jaramillo, J. Bonde, I. Chorkendorff, and J. K. Nørskov, Nature Materials 5, 909 (2006).

${ }^{10}$ F. Studt, F. Abild-Pedersen, T. Bligaard, R. Z. Sorensen, C. H. Christensen, and J. K. Norskov, Science 320, 1320 (2008)

${ }^{i 1}$ E. Skúlason, T. Bligaard, S. Gudmundsdóttir, F. Studt, J. Rossmeisl, F. Abild-Pedersen, T. Vegge, H. Jónsson, and J. K. Nørskov, Phys. Chem. Chem. Phys. 14, 1235 (2012)

${ }^{12}$ L. R. Johnson, S. Sridhar, L. Zhang, K. D. Fredrickson, A. S. Raman, J. Jang, C. Leach, A. Padmanabhan, C. C. Price, N. C. Frey, A. Raizada, V. Rajaraman, S. A. Saiprasad, X. Tang, and A. Vojvodic, ACS Catalysis 10, 253 (2020)

${ }^{13}$ G. Hautier, A. Jain, H. Chen, C. Moore, S. P. Ong, and G. Ceder, Journal of Materials Chemistry 21, 17147 (2011)

${ }^{14}$ S. Curtarolo, W. Setyawan, G. L. Hart, M. Jahnatek, R. V. Chepulskii, R. H. Taylor, S. Wang, J. Xue, K. Yang, O. Levy, M. J. Mehl, H. T. Stokes, D. O. Demchenko, and D. Morgan, Computational Materials Science 58, 218
(2012)

is P. H. Dederichs and R. Zeller, Physical Review B 28, 5462 (1983).

16 G. P. Kerker, Physical Review B 23, 3082 (1981)

17 D. Raczkowski, A. Canning, and L. W. Wang, Physical Review B 64, 121101 (2001).

18 Y. Zhou, H. Wang, Y. Liu, X. Gao, and H. Song, Physical Review E 97, 033305 (2018).

19 L. Lin and C. Yang, SIAM Journal on Scientific Computing 35, S277 (2013).

20 P. Hasnip and M. Probert, arXiv preprint arXiv:1503.01420 (2015).

21 K.-M. Ho, J. Ihm, and J. D. Joannopoulos, Physical Review B 25, 4260 (1982).

22 P.-M. Anglade and X. Gonze, Physical Review B 78, 045126 (2008).

23 N. Woods, M. Payne, and P. Hasnip, Journal of Physics: Condensed Matter 31, 453001 (2019).

24 X. Gonze, Physical Review B 54, 4383 (1996).

25 E. Cancès, G. Kemlin, and A. Levitt, arXiv preprint arXiv:2004.09088 (2020).

${ }^{26}$ M. Chupin, M.-S. Dupuy, G. Legendre, and É. Séré, arXiv preprint arXiv:2002.12850 (2020).

27 Y. Saad, Iterative Methods for Sparse Linear Systems, 2nd ed., edited by Y. Saad (SIAM Publishing, 2003).

28 A. Levitt, Archive for Rational Mechanics and Analysis , 1 (2020).

29 É. Cancès and M. Lewin, Archive for Rational Mechanics and Analysis 197, 139 (2010).

30 R. Resta, Physical Review B 16, 2717 (1977).

31 S. Kumar, Q. Xu, and P. Suryanarayana, Chemical Physics Letters 739, 136983 (2020).

32 M. F. Herbst and A. Levitt, "Density-functional toolkit (DFTK), version v0.1.8," (2020), https://dftk.org

33 J. P. Perdew, K. Burke, and M. Ernzerhof, Physical Review Letters 77, 3865 (1996)

34 S. Lehtola, C. Steigemann, M. J. Oliveira, and M. A. Marques, SoftwareX 7, 1 (2018).

35 S. Goedecker, M. Teter, and J. Hutter, Physical Review B 54, 1703 (1996).

${ }^{36}$ M. F. Herbst and A. Levitt, "Computational scripts and raw data for the presented study of preconditioners," https://github.com/mfherbst/ 
supporting-ldos-preconditioning

H. F. Wilson, F. Gygi, and G. Galli, Physical Review B 78 (2008), 10.1103/physrevb.78.113303

${ }^{38}$ H. F. Wilson, D. Lu, F. Gygi, and G. Galli, Physical Review B 79 (2009), 10.1103/physrevb.79.245106

39 E. Cancès and V. Ehrlacher, Archive for rational mechanics and analysis 202, 933 (2011).

40 P. Giannozzi, S. Baroni, N. Bonini, M. Calandra, R. Car, C. Cavazzoni, D. Ceresoli, G. L. Chiarotti, M. Cococcioni, I. Dabo, A. Dal Corso, S. de Gironcoli, S. Fabris, G. Fratesi, R. Gebauer, U. Gerstmann, C. Gougoussis, A. Kokalj, M. Lazzeri, L. Martin-Samos, N. Marzari, F. Mauri, R. Mazzarello, S. Paolini, A. Pasquarello, L. Paulatto, C. Sbraccia, S. Scandolo, G. Sclauzero, A. P. Seitsonen, A. Smogunov, P. Umari, and R. M. Wentzcovitch, Journal of Physics: Condensed Matter 21, 395502
(2009)

${ }^{41}$ P. Giannozzi, O. Andreussi, T. Brumme, O. Bunau, M. Buongiorno Nardelli, M. Calandra, R. Car, C. Cavazzoni, D. Ceresoli, M. Cococcioni, N. Colonna, I. Carnimeo, A. Dal Corso, S. de Gironcoli, P. Delugas, R. A. DiStasio, A. Ferretti, A. Floris, G. Fratesi, G. Fugallo, R. Gebauer, U. Gerstmann, F. Giustino, T. Gorni, J. Jia, M. Kawamura, H.-Y. Ko, A. Kokalj, E. Küçükbenli, M. Lazzeri, M. Marsili, N. Marzari, F. Mauri, N. L. Nguyen, H.V. Nguyen, A. Otero-de-la Roza, L. Paulatto, S. Poncé, D. Rocca, R. Sabatini, B. Santra, M. Schlipf, A. P. Seitsonen, A. Smogunov, I. Timrov, T. Thonhauser, P. Umari, N. Vast, X. Wu, and S. Baroni, Journal of Physics: Condensed Matter 29, 465901 (2017)

4 J. Sipe and J. Van Kranendonk, Molecular Physics 35, 1579 (1978). 